\section{BMJ Open Respiratory Research}

\title{
Use of bubble continuous positive airway pressure (bCPAP) in the management of critically ill children in a Malawian paediatric unit: an observational study
}

\author{
Sarah Myers, ${ }^{1}$ Precious Dinga, ${ }^{2}$ Margot Anderson, ${ }^{3,4}$ Charles Schubert, ${ }^{5,6}$ \\ Rachel Mlotha, ${ }^{2}$ Ajib Phiri, ${ }^{4}$ Tim Colbourn, ${ }^{7}$ Eric Douglass McCollum, ${ }^{8}$ \\ Charles Mwansambo, ${ }^{9}$ Peter Kazembe, ${ }^{10}$ Hans-Joerg Lang ${ }^{4,11}$
}

To cite: Myers S, Dinga $P$, Anderson M, et al. Use of bubble continuous positive airway pressure (bCPAP) in the management of critically ill children in a Malawian paediatric unit: an observational study. BMJ Open Resp Res 2019;6:e000280. doi:10.1136/ bmjresp-2018-000280

Received 12 February 2018 Revised 27 October 2018 Accepted 16 November 2018

Check for updates

C) Author(s) (or their employer(s)) 2019. Re-use permitted under CC BY-NC. No commercial re-use. See rights and permissions. Published by BMJ.

For numbered affiliations see end of article.

Correspondence to Dr Hans-Joerg Lang; hajoe123@yahoo.es

\section{ABSTRACT}

Introduction In low-resource countries, respiratory failure is associated with a high mortality risk among critically ill children. We evaluated the role of bubble continuous positive airway pressure (bCPAP) in the routine care of critically ill children in Lilongwe, Malawi. Methods We conducted an observational study between 26 February and 15 April 2014, in an urban paediatric unit with approximately 20000 admissions/ year (in-hospital mortality $<5 \%$ approximately during this time period). Modified oxygen concentrators or oxygen cylinders provided humidified bCPAP air/oxygen flow. Children up to the age of 59 months with signs of severe respiratory dysfunction were recruited. Survival was defined as survival during the bCPAP-treatment and during a period of 48 hours following the end of the bCPAP-weaning process.

Results 117 children with signs of respiratory failure were included in this study and treated with bCPAP. Median age: 7 months. Malaria rapid diagnostic tests were positive in $25(21 \%)$ cases, $15(13 \%)$ had severe anaemia $(\mathrm{Hb}<7.0 \mathrm{~g} / \mathrm{dL}) ; 55$ (47\%) children had multiorgan failure (MOF); 22 (19\%) children were HIVinfected/exposed. 28 (24\%) were severely malnourished. Overall survival was 79/117 (68\%); survival was 54/62 $(87 \%)$ in children with very severe pneumonia (VSPNA) but without MOF. Among the 19 children with VSPNA (single-organ failure (SOF)) and negative HIV tests, all children survived. Survival rates were lower in children with MOF (including shock) (45\%) as well as in children with severe malnutrition (36\%) and proven HIV infection or exposure (45\%).

Conclusion Despite the limitations of this study, the good outcome of children with signs of severe respiratory dysfunction (SOF) suggests that it is feasible to use bCPAP in the hospital management of critically ill children in resource-limited settings. The role of bCPAP and other forms of non-invasive ventilatory support as a part of an improved care package for critically ill children with MOF at tertiary and district hospital level in low-resource countries needs further evaluation. Critically ill children with nutritional deficiencies and/or HIV infection/exposure need further study to determine bCPAP efficacy.

\section{Key messages}

The high survival rates among children with very severe pneumonia and no other severe organ dysfunctions, documented in this study, indicate that bCPAP can be used safely and efficiently in resource-limited but well-supervised paediatric units in SSA.

- Multiorgan failure, malnutrition and HIV infection/exposure were associated with an increased mortality risk. The role of bCPAP and other forms of NIV as a part of an improved care package for malnourished and non-malnourished critically ill children (including shock \pm anaemia) needs further evaluation.

- The study highlights the importance of a multidisciplinary approach in the implementation process of basic paediatric critical care measures like bCPAP e.g.: efficient logistic set-up of patient circuits, reliable oxygen systems including reliable electricity supply, cost-effective, good quality medical equipment, biomedical input, support of health workers and training.

\section{INTRODUCTION}

In $2015,5.9$ million children died before the age of 5 years with approximately $50 \%$ of these deaths occurring in sub-Saharan Africa (SSA). ${ }^{1}$ Very severe pneumonia (VSPNA) based on the WHO classification remains a leading cause of child mortality. ${ }^{1}$ Although there has been a considerable decline in mortality in hospitalised Malawian children between 2000 and 2012, mortality remained high in 2012 in critical subgroups including those with VSPNA (12\%), severe undernutrition $(15 \%)$ and severe acute malnutrition $(35 \%) .^{2}$ Despite the introduction of Haemophilus influenzae $\mathrm{B}$ and pneumococcal conjugate vaccines, severe respiratory infections (bacterial and viral) are likely to remain a major cause of morbidity and mortality in 
SSA. Paediatric emergency and critical care, adapted to available resources, is therefore essential in order to achieve further reductions in child mortality. Improvements of non-invasive respiratory support can play an important role in this context.

Respiratory failure is also a common feature of critically ill children with severe sepsis. ${ }^{3}$ Severe infections can lead to a complex systemic inflammatory response, and a variety of conditions such as pneumonia, bacteraemia and malaria can coexist. ${ }^{45}$ Without early, efficient management, these infections can progress to significant organ dysfunction, including acute respiratory distress syndrome and respiratory failure. ${ }^{3}$ Respiratory support is therefore an essential element in the management of critically ill, septic children. ${ }^{6-8}$

Improved paediatric emergency care in health facilities and streamlined referral pathways can have a significant impact on child survival. Improvement of oxygen delivery and respiratory support plays an important role in this context. A recent study conducted in Malawi showed that the use of pulse oximetry in peripheral outpatient health facilities and village clinics can help clinicians and community health workers to identify children with severe pneumonia and hypoxaemia. ${ }^{9}$ A study in Papua New Guinea demonstrated that the reliable supply of low flow oxygen can reduce mortality among children with signs of severe pneumonia. ${ }^{10}$ Oxygen concentrators in conjunction with reliable electricity systems associated with 'uninterruptable power supply' are cost-effective and provide a reliable method of oxygen supply in low-resource settings. ${ }^{11}{ }^{12}$ Installation and maintenance of these 'oxygen systems', however, remain a challenge in many health facilities in African countries. ${ }^{13}$ The combination of solar-powered energy systems and the use of oxygen concentrators can be an option to improve oxygen supply in health facilities in remote settings. ${ }^{14}$

Non-invasive respiratory support has the potential to improve the outcome of critically ill children managed in low-resource settings, and notably, the role of non-invasive ventilatory (NIV) support in the management of septic children with 'less severe Acute Respiratory Distress Syndrome (ARDS)' is also under evaluation in high-resource settings. ${ }^{15}$

Different forms of NIV exist: continuous positive airway pressure (CPAP), high-flow of warmed and humidified air/oxygen via nasal cannula, bilevel positive airway pressure and neuronally adjusted ventilatory assist. ${ }^{16} 17$

CPAP has been used efficiently for the treatment of children with respiratory dysfunction in high-resource settings (eg, viral bronchiolitis). ${ }^{18-20}$ It has been shown that bubble CPAP (bCPAP) administered through nasal prongs offers a good quality, relatively low-cost alternative to ventilator-delivered CPAP on neonatal units. ${ }^{21}$ Feasibility and safety of bCPAP use on neonatal units in middle-income and low-income countries have also been evaluated. ${ }^{22}$

A recent randomised controlled trial (RCT) conducted in Bangladesh showed that children with VSPNA treated with bCPAP had a significantly lower mortality rate $(4 \%)$ compared with a group treated with low-flow $\mathrm{O}_{2}(15 \%){ }^{23}$ A further study conducted in Ghana demonstrated that CPAP can be used efficiently in the management of children with respiratory distress in SSA. ${ }^{24}$

A smaller study described the use of an improvised bCPAP set-up (mostly non-humidified flow) on an extremely busy paediatric unit in Malawi for children (29 days to 59 months) with VSPNA and children presenting with signs of respiratory dysfunction in the context of critical illness (eg, severe malaria) including shock and severe anaemia. ${ }^{25}$ Several months later, the authors had the opportunity to introduce a better adapted bCPAP set-up in the same clinical environment. Its use with a larger and better-described cohort, and its success or failure dependent on patient risk characteristics and their association with death while on bCPAP, is reported here.

\section{METHODS}

\section{Study population and setting}

We conducted an observational study of all children $0-15$ years treated with bCPAP between 26 February and 15 April 2014 in the paediatric department at Kamuzu Central Hospital in Lilongwe, Malawi. In 2014, this department had approximately 20000 paediatric admissions of infants and children ages $0-15$ years. This observational study was conducted during the peak of the malaria season in 2014, with $>3000$ admissions / month in March and April 2014 and a mortality rate of approximately $4 \% .{ }^{26}$ Most newborns were treated on a dedicated neonatal unit. A small number of newborns born outside Kamuzu Central Hospital, received respiratory support with bCPAP on the paediatric unit. Only these newborns were included in the study.

Patients were initially screened in a triage/admission zone. Critically ill children with emergency signs ${ }^{27}$ were managed in a 'resuscitation zone (4 beds for 4 , sometimes up to 16 patients)'. This area was directly connected to a 'critical care/emergency zone' (approximately $16-18$ beds-for up to 30-60 patients), where further basic critical care, according to WHO guidelines $^{27}$ (including management of airway and breathing, intravenous or intraosseous fluids, blood transfusion, nutritional support, oral and intravenous medications), was provided. Approximately eight oxygen concentrators, backed up with oxygen cylinders, were available. The oxygen flow provided by each of these devices can be split to deliver low-flow $\mathrm{O}_{2}$ to up to five patients.

This centralisation of basic critical care enabled a limited number of staff to provide the best possible management (including vital signs approximately 2-3 times per day and more frequent monitoring in very sick patients) to a large number of critically ill children (nurse to patient ratio: 1:15-30, doctors/clinical officers: 2-4 (day) and 1-2 (night); supervision by at least one consultant on site for 12 hours per day). In a six-bed 'high 
dependency unit (HDU)', closer observation of selected critically ill children could be provided (spot-checks of vital signs approximately 2-4 hourly). The number of HDU beds was insufficient for the management of the large number of critically ill children.

Survival was defined as survival during the bCPAP treatment and during a period of 48 hours following the end of the bCPAP-weaning process.

The unit's policy was to offer HIV testing and counselling to every hospitalised child. However, because of the high workload and limited numbers of HIV counsellors not all admitted children were tested prior to death or discharge.

Basic 'point of care tests' were usually available, including malaria rapid diagnostics tests (MRDT), haemoglobin tests and blood sugar analysis. Further laboratory tests like blood gases, electrolytes, renal and liver function, and full blood counts were not available. The blood bank was able to provide urgent whole blood transfusions generally within 2-4 hours but at times there was more of a delay.

Basic bedside ultrasound and echocardiography helped to define thoracic and abdominal pathologies, cardiac function and congenital heart diseases. Access to chest X-rays was inconsistent.

\section{Definitions and comorbidities}

VSPNA was diagnosed by the presence of cough or difficulty breathing with either $\mathrm{SpO}_{2}<90 \%$, central cyanosis, severe respiratory distress (very severe chest indrawing), danger signs such as inability to drink or breast feed, lethargy, and reduced level of consciousness. Multiorgan failure (MOF) was defined as having respiratory dysfunction with either cardiovascular dysfunction (including severe anaemia) or central nervous system dysfunction at the time of bCPAP initiation. Severe anaemia was defined as an $\mathrm{Hb}<7 \mathrm{~g} / \mathrm{dL}$ or clinically when rapid point-of-care testing was not available. Central nervous system dysfunction was defined as a Blantyre Coma Scale $\leq 3$ or status epilepticus.

HIV status was defined as 'infected' if a child $<2$ years had a positive DNA PCR test or a child $\geq 2$ years had a positive rapid test. A child was considered to be HIV 'exposed' if there was a positive rapid test for a child $<2$ years or a documented maternal positive test, but no DNA PCR had yet been performed.

Malaria was diagnosed by MRDT, and all children with malaria receiving bCPAP would have been found to have severe malaria by virtue of their respiratory distress, shock or neurological dysfunction.

Nutritional status was described as either normal, moderate acute malnutrition or severe acute malnutrition by WHO guidelines when data-usually mid-upper arm circumference (MUAC) — were available.

Other comorbidities included congenital heart defects diagnosed by bedside echocardiography, cerebral palsy, spina-bifida, hydrocephalus, neuromuscular disorders and other forms of neurodisability, sickle cell disease, malignancy or clinically suspected tuberculosis.

\section{Inclusion criteria and initiation of bCPAP}

Critically ill children of any age were initiated on bCPAP if, after initial resuscitation, they were found to have severe respiratory distress with or without further organ dysfunctions like shock, severe anaemia or neurological dysfunction. bCPAP could be initiated in the emergency zone or HDU depending on bed availability.

\section{Fluid resuscitation}

Children with signs of shock and no signs of dehydration received only careful fluid resuscitation $(10 \mathrm{~mL} /$ $\mathrm{kg}$ isotonic crystalloid solution). After re-evaluation, one further fluid bolus was administered in some children (a third bolus was only given very rarely). Malnourished children with signs of shock and no dehydration received only one fluid bolus. If possible, haemoglobin $(\mathrm{Hb})$ was rechecked after each fluid bolus.

Children with severe anaemia did not receive a pre-transfusion bolus. In case of severe anaemia, $20 \mathrm{~mL} /$ $\mathrm{kg}$ whole blood was transfused over 3-4 hours if HBs were $<5-6 \mathrm{~g} / \mathrm{dL}$. This practice is in line with the latest WHO recommendations from 2016. ${ }^{28}$

In case of severe dehydration, WHO guidelines for the management of malnourished and non-malnourished children were followed as well. ${ }^{28}$

\section{bCPAP device (see figure 1)}

The low-cost bCPAP devices used in this study were modified oxygen concentrators with a maximum air/oxygen flow of 16 litres per min (LPM) (maximum oxygen flow: 8 LPM, maximum air flow: 8 LPM; http:/ /go.gomango. co.uk/diamedica.co.uk/english/product_details_ diamedica.cfm?id=1562). The $\mathrm{FiO}_{2}$ could be determined by adjusting the ratio of oxygen/air flow. The blended flow was humidified and in some devices could be warmed using the heat produced by the compressor of the oxygen concentrator. The pressure was determined by the distance of the distal part of the expiratory limb of the ventilation circuit below the water surface of a water bottle ('resistor').

In case not enough 'bCPAP set-ups' were available, oxygen cylinders were used to provide $\mathrm{O}_{2}$ flow required to provide bCPAP. With this 'suboptimal set-up', the $\mathrm{FiO}_{2}$ could not be adapted but oxygen flow could still be humidified. Patients were switched to the described 'modified oxygen concentrator' whenever such a device was available.

\section{Basic further care of critically ill children managed with bCPAP}

Additional aspects of care for patients on bCPAP included head-up positioning, nasogastric tube (NGT) insertion for initial gastric decompression and drainage, intravenous maintenance fluids initially at $2 / 3$ of the 


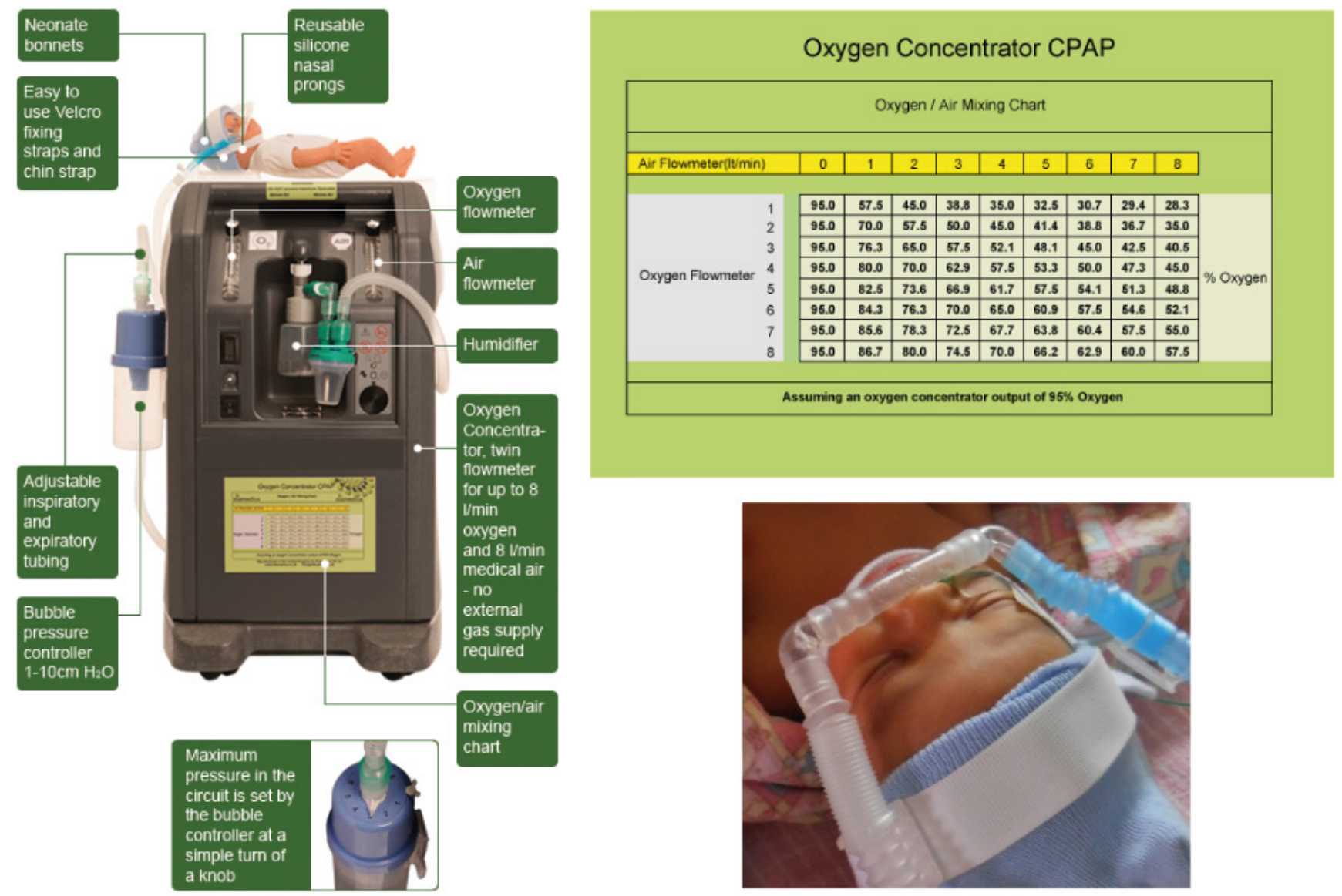

Figure 1 Bubble continuous positive airway pressure (bCPAP) set-up. (With permission of Diamedica: http://go.gomango. co.uk/diamedica.co.uk/english/product_details_diamedica.cfm?id=1562)

'4-2-1 rule'29 were administered. Isotonic electrolyte solutions (Ringers lactate and dextrose) ${ }^{30}$ were used as maintenance fluids. Once vital organ functions were stabilised, 3-hourly NGT feeds were introduced gradually with expressed breast milk for infants, F-75 for children $>6$ months with SAM. Regarding enteral nutrition of critically ill children without severe acute malnutrition (SAM): Not enough therapeutic nutrition (F75 or F100) was available for the nutrition of critically ill children without SAM. No other specific enteral nutrition was available for this purpose. Therefore ordinary milk products or porridge were used for gastric tube feeding of these critically ill children. In many cases, tolerance of enteral feeds was initially tested with the administration of oral rehydration fluids via NGTs. The maximum volume of enteral feeds administered during this 'critical care phase' for malnourished and non-malnourished children was $100 \%$ according to the "4-2-1 rule,"29.

National and WHO guidelines were applied to treat tuberculosis, HIV, malnutrition and common conditions like severe malaria, respiratory infections, diarrheal diseases, meningitis and bacterial sepsis. ${ }^{27}$

\section{Monitoring and vital signs}

Rapid clinical screening was done to triage patients requiring non-invasive respiratory support. Basic vital signs (heart rate $(\mathrm{HR})$, respiratory rate (RR), oxygen saturation $\left(\mathrm{SpO}_{2}\right)$, temperature) were monitored usually 2-3 times per day by vital signs assistants ${ }^{31}$ or qualified nurses.

\section{CPAP settings}

bCPAP pressure was commenced at $6-7 \mathrm{~cm} \mathrm{H}_{2} \mathrm{O} .{ }^{19}$ The initial $\mathrm{FiO}_{2}$ was set at approximately $0.6 \%$. Pressure and $\mathrm{FiO}_{2}$ were adjusted depending on signs of work of breathing and peripheral oxygen saturation measurement by pulse oximetry $\left(\mathrm{SpO}_{2}\right)$. The aim was to maintain $\mathrm{SpO}_{2}>94 \%$. bCPAP pressure was usually kept $\leq 8 \mathrm{~cm} \mathrm{H}_{2} \mathrm{O}$ and only in rare cases increased to $10 \mathrm{~cm} \mathrm{H}_{2} \mathrm{O}$. Humidified air/oxygen flow was used for all children.

\section{bCPAP weaning protocol}

When using the modified oxygen concentrator set-up, $\mathrm{FiO}_{2}$ was reduced when $\mathrm{SpO}_{2}$ was consistently $>94 \%$ (FIO ${ }_{2}$ could not be adapted if $\mathrm{O}_{2}$ cylinders were used as 
flow generator). If work of breathing and RR improved with $\mathrm{SpO}_{2}$ remaining consistently $>94 \%$ (with an $\mathrm{FIO}_{2}$ $\leq 0.5 \%$ ) bCPAP pressures were reduced gradually. If the patient remained stable and the bCPAP level was $\leq 5 \mathrm{~cm}$ $\mathrm{H}_{2} \mathrm{O}$ (with an $\mathrm{FIO}_{2}<0.3-0.4$ ), then a trial 'off bCPAP' was started and the child was placed on low-flow oxygen (1-2 LPM). In cases of clinical deterioration, bCPAP was restarted. This clinical scenario was not considered as a 'treatment failure'. In the data analysis, this additional phase of 'bCPAP management' was considered as part of the 'bCPAP weaning process' and the initial treatment. The duration of the weaning process was depending on the child's clinical progress. In the data analysis, the end of the weaning process was documented as the end of the bCPAP treatment.

\section{Data collection and analysis}

A case record form, an observation chart and a patient register were used for data collection. Data were entered into an Excel database. We describe the proportion of bCPAP cases who survived by risk group, sex, age, complications, HIV status and treatment methods. We conducted univariable logistic regression to determine if the odds of bCPCP survival were higher in particular subgroups. The small number of cases precluded multivariable analysis. We also present a breakdown of survival and death (failure) by HIV status and comorbidities including shock, malaria, anaemia, MOF and severe malnutrition, and describe the reasons for complications of treatment. This study was done in a routine setting without dedicated funding and did not have a target sample size. The sample includes most bCPAP cases seen between 26 February and 15 April 2014. All analyses were done in Stata V.14.2 for Mac.

\section{RESULTS}

A total of 117 patients were included and $79(68 \%)$ survived (table 1). Survival was similar among males and females, and did not differ statistically by age group, though there were small sample sizes in each group. Survival was lower in those with MOF (45\%) than those with single-organ failure (SOF) and no comorbidities (86\%; $\mathrm{p}<0.0001$, table 1$)$. Survival was also significantly lower in those with severe acute malnutrition $(36 \%)$ than with no severe or moderate malnutrition $(81 \%$; table 1$)$, and in those with confirmed HIV infection or exposure $(45 \%)$ than those who were HIV negative $(81 \%)$ or with an unknown HIV status (68\%; table 1). There were no statistically significant differences in survival by bCPAP delivery device or treatment initiation location (table 1).

All 19 children with VSPNA who were HIV negative and had no comorbidities and no other organ dysfunctions survived (five children in this group had suspected TB). Also, $40 / 42$ (95\%; $95 \%$ CI $84 \%$ to $99 \%)$ of children with VSPNA (unknown or negative HIV status) and no comorbidities and no other organ dysfunction survived. Children with VSPNA and HIV infection/exposure and/ or malnutrition had significantly increased mortality risk (see table 2). Respiratory failure with additional organ dysfunctions (shock, anaemia) was associated with lower survival rates.

In total, 14/15 (93\%) children with severe anaemia were transfused. Four out of seven with severe anaemia and shock survived. Also, 6 out of 14 children with anaemia who were transfused died. The one patient with severe anaemia who was not transfused survived. And, 11 out of 15 children with severe anaemia had positive malaria tests. Also, 7 of these 11 children survived.

Among the 31 children with severe malaria, 24 had multiorgan dysfunction. In total, 14 of these 24 children survived. Of children with positive malaria tests, 11/31 $(35 \%)$ had signs of shock. Also, 5 of these 11 children survived.

\section{Technical issues and complications}

The average flow set was 9.1 LPM (SD 2.0, range 5-16). The average starting pressure was $6.7 \mathrm{~cm} \mathrm{H}_{2} \mathrm{O}$ (SD 1.0, range 4-9).

In total, $13 / 117$ patients experienced equipment-related complications, including blocked nostrils (2) or nasal prongs (2), interruption of oxygen supply from depleted cylinder (2), nasal septum lesions (7) and aspiration of feeds (1). One parent refused the continuation of bCPAP. Some patients had more than one complication. The total number of treatment failures among those experiencing complications was $3 / 13$ patients.

\section{DISCUSSION}

This basic observational study was conducted on a very busy paediatric unit in Malawi during peak malaria season.

We describe a group of children with a high mortality risk. Because of resource limitations, we took the approach of offering bCPAP to those children having the most severe clinical presentations.

In high-resource settings, a considerable number of the 62 children with respiratory dysfunction and the 55 children with MOF potentially would have been mechanically ventilated and would have received further critical care interventions in a paediatric intensive care unit (PICU). ${ }^{7}$

Context-adapted disease severity scores (comparable to Paediatric Index of Mortality (PIM) ${ }^{32} /$ Pediatric Risk of Mortality Score (PRISM) ${ }^{33}$-scores used in PICUs) based on parameters describing acute physiological status and underlying conditions (eg, HIV infection, nutritional status) can help to describe and compare cohorts of critically ill children and adults managed in low-resource settings. Further research in this context is needed. ${ }^{34-37}$

In the study setting, only some children could benefit from intubation and ventilation. Surveys highlight the challenges associated with the set-up of intensive care units providing mechanical ventilation of children. Significant resources will be directed to only a small 
Table 1 Demographics and outcomes

\begin{tabular}{|c|c|c|c|c|}
\hline & Patients (n) & $\begin{array}{l}n=\text { survived bCPAP } \\
(\%)^{\star}\end{array}$ & $\begin{array}{l}\mathrm{n}=\mathrm{bCPAP} \text { death } \\
(\%)^{*}\end{array}$ & OR of death $(95 \% \mathrm{Cl})$ \\
\hline Total & 117 & $79(68 \%)$ & $38(32 \%)$ & \\
\hline \multicolumn{5}{|l|}{ Demographics } \\
\hline Male† & 58 & $40(69 \%)$ & $18(31 \%)$ & 1.00 \\
\hline Female & 51 & $34(67 \%)$ & $17(33 \%)$ & $1.11(0.50$ to 2.49$)$ \\
\hline Median (IQR) age in months & $7(2-15)$ & $6(2-15)$ & $7(4-14)$ & \\
\hline \multicolumn{5}{|l|}{ Age (months) $\dagger$} \\
\hline$<1$ & 6 & $3(50 \%)$ & $3(50 \%)$ & 1.00 \\
\hline $1-12$ & 76 & $53(70 \%)$ & $23(30 \%)$ & $0.43(0.81$ to 2.31$)$ \\
\hline $13-24$ & 23 & $17(74 \%)$ & $6(26 \%)$ & 0.35 (0.06 to 2.25$)$ \\
\hline $25-48$ & 10 & $5(50 \%)$ & $5(50 \%)$ & $1.00(0.13$ to 7.57$)$ \\
\hline \multicolumn{5}{|l|}{ Organ failure } \\
\hline VSPNA & 62 & $54(87 \%)$ & $8(13 \%)$ & \\
\hline $\begin{array}{l}\text { VSPNA no comorbiditiesł; including } \\
\text { children<1 month }\end{array}$ & 59 & $51(86 \%)$ & $8(14 \%)$ & 1.00 \\
\hline VSPNA with comorbidities $\ddagger$ & 3 & $3(100 \%)$ & $0(0 \%)$ & \\
\hline $\begin{array}{l}\text { VSPNA no comorbidities; no HIV } \\
\text { exposure/infection, no malaria, no } \\
\text { suspected TB }\end{array}$ & 14 & $14(100 \%)$ & $0(0 \%)$ & \\
\hline $\begin{array}{l}\text { VSPNA; no comorbidities; no HIV } \\
\text { exposure/infection, no malaria; } \\
\text { including suspected TB }\end{array}$ & 19 & $19(100 \%)$ & $0(0 \%)$ & \\
\hline $\begin{array}{l}\text { MOF >1 organ dysfunction } \neq: \\
\text { respiratory dysfunction and at least } \\
\text { one further organ dysfunction }\end{array}$ & 55 & $25(45 \%)$ & $30(55 \%)$ & $\begin{array}{l}\text { vs VSPNA: } 8.10 \text { (3.25 to } \\
20.18) \emptyset\end{array}$ \\
\hline $\begin{array}{l}\text { MOF > } 2 \text { organ dysfunctionsł: } \\
\text { (respiratory dysfunction and at least } \\
\text { two other organ dysfunctions) }\end{array}$ & 21 & $9(43 \%)$ & $12(57 \%)$ & \\
\hline \multicolumn{5}{|l|}{ Malnutrition $†$} \\
\hline SAM & 28 & $10(36 \%)$ & $18(64 \%)$ & 7.59 (2.88 to 19.97$) \S$ \\
\hline MAM & 13 & $8(62 \%)$ & $5(38 \%)$ & 2.63 (0.75 to 9.29$)$ \\
\hline No MAM or SAM & 73 & $59(81 \%)$ & $14(19 \%)$ & 1.00 \\
\hline \multicolumn{5}{|l|}{ HIV status } \\
\hline Infected or exposed & 22 & $10(45 \%)$ & $12(55 \%)$ & 4.97 (1.53 to 16.13$)$ ๆ \\
\hline Negative & 36 & $29(81 \%)$ & $7(19 \%)$ & 1.00 \\
\hline Unknown & 59 & $40(68 \%)$ & $19(32 \%)$ & 1.97 (0.73 to 5.29$)$ \\
\hline \multicolumn{5}{|l|}{ bCPAP delivery device } \\
\hline Cylinder & 50 & 35 (70\%) & $15(30 \%)$ & 1.00 \\
\hline Concentrator & 48 & $30(62 \%)$ & $18(38 \%)$ & 1.40 (0.60 to 3.25$)$ \\
\hline Unrecorded & 17 & $12(71 \%)$ & $5(29 \%)$ & 0.97 (0.29 to 3.25$)$ \\
\hline \multicolumn{5}{|l|}{ Treatment initiation location } \\
\hline High dependency unit & 29 & 17 (59\%) & $12(41 \%)$ & 1.55 (0.65 to 3.70$)$ \\
\hline Emergency zone & 83 & 57 (69\%) & $26(31 \%)$ & 1.00 \\
\hline \multicolumn{5}{|l|}{ Intubation** } \\
\hline No & 100 & 77 (77\%) & $23(23 \%)$ & 1.00 \\
\hline Yes & 15 & $2(13 \%)$ & $13(87 \%)$ & 21.8 (4.6 to 103.5$) \dagger \dagger$ \\
\hline
\end{tabular}




\begin{tabular}{lllll}
\hline & Patients (n) & $\begin{array}{l}\mathbf{n = s u r v i v e d ~ b C P A P} \\
(\%)\end{array}$ & $\begin{array}{l}\mathbf{n = b C P A P} \text { death } \\
(\%))^{*}\end{array}$ & OR of death (95\% CI) \\
\hline $\begin{array}{l}\text { Median (IQR) treatment duration } \\
\text { (hours) }\end{array}$ & $24(24-60)$ & $24(24-60)$ & $24-48)$ \\
\hline
\end{tabular}

Comorbidities: congenital heart diseases (4 (children with congenital heart diseases: AVSD: 2 (1 with trisomy 21), VSD: 1; PDA: 1 (and trisomy 21))), neurodisability (cerebral palsy (1), hydrocephalus (1), neuromuscular disorder (1), severe thorax wall infection/defect (1). bCPAP,bubble continuous positive airway pressure; HB, haemoglobin; MAM, moderate acutemalnutrition; MOF, multiorgan failure; SAM, severe acute malnutrition; TB,tuberculosis; VSPNA, very severe pneumonia. AVSD, Atrio-ventricular Septum Defect; VSD, Ventricular Septum Defect; PDA, Persistent Ductus Arteriosus

\footnotetext{
*The percentages given in column 3 and 4 describe the ratios to the subtotals documented in column 2.

†Information on sex (male/female) was not recorded for eight patients. Information on age was not recorded for two patients. Information on malnutrition (MAM or SAM) was not recorded for three patients.

$\ddagger$ Organ dysfunction: respiratory failure (signs of very severe pneumonia ${ }^{50}$, signs of shock or 'impaired circulation, ${ }^{, 28}$, severe anaemia $(\mathrm{HB}<7$ $\mathrm{g} / \mathrm{dl})$, coma/prolonged convulsions, clinical signs of liver/renal failure.

$\S \mathrm{p}<0.0001$.

Ip $=0.008$.

${ }^{*}$ In 2 of the 117 patients the information on potential intubation and ventilation was not documented. $\dagger \dagger p<0.000$.

bCPAP, bubble continuous positive airway pressure; HB, haemoglobin; MAM, moderate acutemalnutrition; MOF, multiorgan failure; SAM, severe acute malnutrition; TB, tuberculosis; VSPNA, very severe pneumonia.
}

number of critically ill patients and specific skill levels are required ${ }^{38-40}$ Routine mechanical ventilation of critically ill children is therefore not yet a feasible option for a large number of paediatric units in SSA.

A survey conducted on the same unit in 2012 using an improvised bCPAP set-up showed the following survival rates: overall survival-41/77 (53\%), survival among children with VSPNA-19/26 (73\%) and VSPNA in HIV-negative children-14/17 (82\%). ${ }^{25}$

Several factors could explain the improved outcome of patients with respiratory failure managed with bCPAP described in this publication. The disease severity of the cohorts described in this publication and the study from 2012 are difficult to compare. Furthermore, in the 2-year interval between the studies training, senior supervision and paediatric emergency care was improved on the unit in Lilongwe. Introduction of conjugated pneumococcal vaccine in the routine vaccination schedule in Malawi in the time period between 2012 and 2014 could have had an impact on the spectrum of pathogens causing severe respiratory infections and other severe bacterial infections in young children. However, the improved bCPAP set-up including the use of humidified air/oxygen might have contributed to improved outcomes as well. There is considerable evidence indicating that the relatively high air/oxygen flow used in NIV should be 'conditioned (warmed and humidified), ${ }^{41} 42$

The outcome of HIV-negative children with severe respiratory dysfunction (SOF) managed with bCPAP in this study (mortality rate in this subgroup: $0 \%$ ) is comparable to outcomes of children with respiratory failure managed with bCPAP in the context of an RCT recently conducted in Bangladesh (mortality rate: $4 \%$ ). ${ }^{23}$ Outcomes of this subgroup indicate that the use of bCPAP as a form of NIV is feasible on a busy paediatric critical care department in SSA.

In high-resource settings, proactive respiratory support is standard care in the management of multiorgan dysfunction. ${ }^{8}$ In this context, the role of NIV in less severe ARDS requires further evaluation..$^{15}$ In low-resource settings, NIV has the potential to improve respiratory care for critically ill children, where options for safe mechanical ventilation are extremely limited.

An increased mortality risk among children with respiratory dysfunction associated with severe malnutrition and/or HIV infection/exposure is suggested by this survey, as demonstrated in other studies. ${ }^{43}$ In total, 17 out of 28 children with SAM treated in this study had more than one organ dysfunction (eg, shock).

An RCT is currently being conducted in Malawi comparing the use of bubble CPAP and low-flow oxygen in children with respiratory failure with and without further organ dysfunctions, including children with HIV and malnutrition. ${ }^{44}$

Children who had their treatment initiated in the emergency zone had a higher survival rate than children treated in the HDU. This difference was not statistically significant. This 'trend' is likely to be explained by a significant selection bias with much sicker patients being treated in the HDU. The six-bedded HDU was certainly too small for a paediatric unit with around 20 000 admissions per year. Following rapid identification further 'context adapted' critical care management in a 'HDU- setting' is very likely to improve survival chances of critically ill children.

One child in this study suffered an episode of aspiration. A recent study conducted in Australia showed that children requiring CPAP did not have an increased risk 


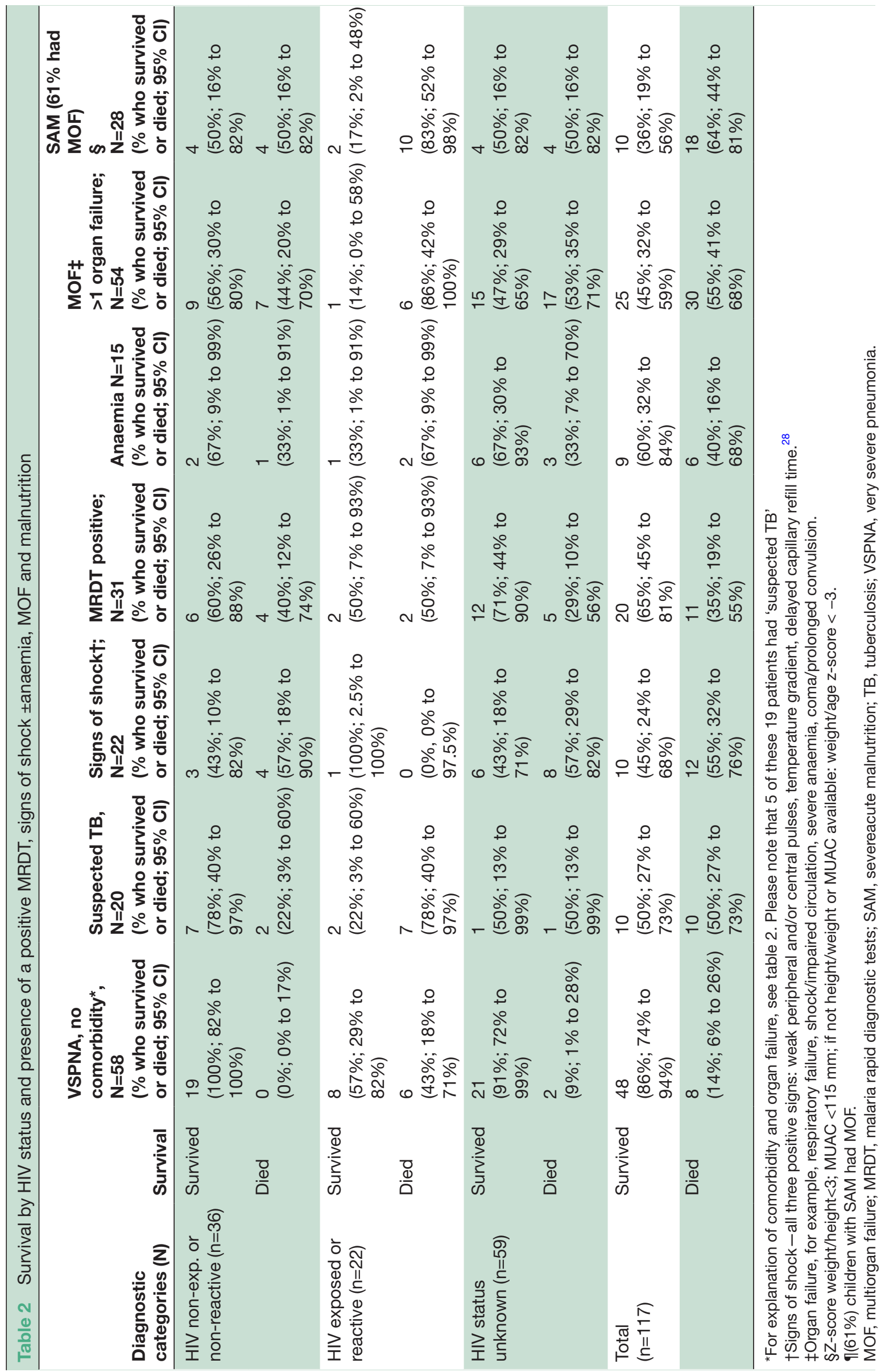


of aspiration, apnoeas/hypoxic events when managed via NGT feeding compared with intravenous maintenance fluids. ${ }^{45}$ Because of limited monitoring options, we could not determine if some of the children had episodes of desaturation associated with NGT feeds. Careful monitoring of maintenance fluids and feeding practices are important elements to consider in the care of critically ill children in low-resource settings. We suggest that introduction of enteral feeds in critically ill malnourished and non-malnourished children managed in low-resource settings should follow basic principles used in paediatric intensive care. Enteral feeds for malnourished and non-malnourished children can be introduced gradually after stabilisation of vital organ functions (eg, stabilisation of respiratory function on $\mathrm{O}_{2}$ or bCPAP, correction of shock, anaemia and dehydration as well as a phase of stability of the child's neurological function, even if still comatosed) and the absence of contraindications (eg, abdominal pathologies, large gastric aspirates).

In total, 11/117 children developed nasal prong-associated problems such as obstruction of the nostrils/nasal prongs (4) or injury of the nasal septum (7). Improved interfaces can help in this context. Good nursing care and adequate nurse to patient ratios in a HDU setting are essential in order to reduce complication rates and achieve good outcomes in critically ill children treated with bCPAP. Efficient and sustained training programmes need to accompany the introduction of bCPAP. Caretakers should be integrated in the care and should be encouraged to notify any concern regarding the condition of their child or any malfunctioning equipment.

Devices used for bCPAP and other forms of NIV in low resources need to be of good quality and should undergo independent biomedical review procedures. ${ }^{46}$ Biomedical repair and maintenance arrangements need to be put in place. Infection prevention and control measures need to be established.

\section{Limitations}

Interpretation of results is limited by the basic observational set-up of the study. The small sample size limited more detailed subgroup analyses. Data quality (eg, frequency of vital signs monitoring) was affected by limited human resources and equipment as well as the extremely high workload. Limited human resources also contributed to the fact that most, but not all, children who were managed with bubble CPAP during the study period were included in the analysis. Some 'CPAP-runs' of short duration might have been missed during weekends. However, we do not think that this contributed to a selection bias, which affected the outcome of the study.

Monitoring of essential elements of critical care management (eg, fluid management) was also affected by low health worker to patient ratio. Adequate numbers of well-trained health workers and basic equipment (eg, $\mathrm{SpO}_{2}$ monitors) can improve care packages and the potential positive impact of bCPAP on the outcome of critically ill patients with respiratory dysfunction. Laboratory tests allowing more detailed evaluation of renal and/ or hepatic dysfunction were not available. This might have contributed to an underestimation of the number of children presenting with MOF. Renal dysfunction is an important parameter determining mortality risk among critically ill children, including children with severe Malaria. ${ }^{47-49}$

Anthropometric measurements were suboptimal in our survey. In many children, we therefore used weight/ age as a marker of nutritional status.

\section{Conclusion}

Despite the limitations of this study, the good outcome of children with signs of VSPNA suggests that it is feasible to use bCPAP in the hospital management of critically ill children in low-resource settings.

BCPAP and other forms of NIV can potentially play an important role in the improvement of care for critically ill children with respiratory failure and further organ dysfunction (eg, shock, severe anaemia) managed in low-resource settings. Further research is required in this context. Children with malnutrition and HIV infection or exposure need particular attention. Different disease processes like malaria, bacterial sepsis and dengue fever need to be considered.

The impact on patient outcome of different forms of NIV should be evaluated. Adapted, good quality equipment with associated maintenance systems is required as well as adequate numbers of health workers and efficient, sustained training activities.

\section{Author affiliations}

${ }^{1}$ South Wales Deanery, Wales, UK

${ }^{2}$ Kamuzu Central Hospital, Lilongwe, Malawi

${ }^{3}$ United States Peace Corps, Washington, DC, USA

${ }^{4}$ Malawian College of Medicine, Lilongwe, Malawi

${ }^{5}$ Department of Pediatrics, University of Cincinnati, College of Medicine, Cincinnati, Ohio, USA

${ }^{6}$ Division of Emergency Medicine, Cincinnati Children Hospital Medical Center, Cincinnati, Ohio, USA

${ }^{7}$ University College London Institute for Global Health, London, UK

${ }^{8} \mathrm{~J}$ ohn Hopkins University School of Medicine, Baltimore, Maryland, USA

${ }^{9}$ Ministry of Health, Lilongwe, Malawi

${ }^{10}$ Baylor College of Medicine, Children Clinical Centre of Excellence, Lilongwe, Malawi

${ }^{11}$ Centre for International Migration and Development (CIM)/German International Cooperation (GIZ), Eschborn, Germany

Acknowledgements The authors acknowledge the support of all the nurses, clinical officers, doctors and support staff working on the paediatric unit at KCH. They also thank all the children admitted to the paediatric unit and their guardians for their understanding and cooperation. They acknowledge the contribution of Paul Sonnenstuhl and Lukas Lang, who supported the data collection and logistic aspects of the study.

Contributors SM: with the support of H-JL she set-up the 'survey structure', including data collection (case record forms, documentation of vital signs and management, etc). She was also involved in the clinical management of patients and clinical training of nurses, clinicians and students. Together with $\mathrm{H}-\mathrm{JL}$ she wrote the initial draft of the manuscript and finalised the publication. PDPD contributed to the nursing care of patients, documentation of patient information, vital signs and management. PD contributed also to practical training of nurses regarding general patient care and specifically on the use of bCPAP. She was of 
great value in the communication with parents and caretakers. MA and CS gave advice on the 'set-up of the survey'. They contributed to the care of patients, training of nurses and clinicians. They also made very important suggestions on the manuscript of the publication. RM advised on the 'set-up' of the survey. She made vital contributions to the introduction to bCPAP on the department's neonatal unit. She was involved in patient care, training of clinicians, nurses and students. RM made important comments on the manuscript of the publication. AP advised on the 'set-up' of the survey. He contributed to patient care and training of health workers and students. AP made important comments on the manuscript of the publication. TC contributed to 'set-up' of the survey regarding rational data collection under the given circumstances. He was responsible for data analysis and contributed to the write-up of the manuscript. EDM contributed to the introduction of bCPAP in Malawi (Lilongwe) and advised on the 'set-up' of the survey. He supported the write-up of the manuscript and interpretation of results. CM advised on the 'set-up' of the survey and supported the survey on administrative level. He made valuable contributions to the manuscript. PK advised on the 'set-up' of the survey and supported the survey on administrative level. He made valuable contributions to the manuscript. He also gave advice on the case management of critically ill children. H-JL contributed to the introduction of bCPAP in Malawi (Blantyre and Lilongwe) and initiated this and a previous survey conducted on the same unit. He contributed to logistic improvements of the department's paediatric emergency unit and the management of critically ill children. He was involved in the training of clinicians, nurses and students. Together with SM he wrote the initial draft of the manuscript and finalised the publication. $\mathrm{H}-\mathrm{JL}$ might include this publication in a portfolio required for an academic degree.

Funding The authors have not declared a specific grant for this research from any funding agency in the public, commercial or not-for-profit sectors.

Competing interests None declared.

Patient consent for publication Not required.

Ethics approval Malawian National Health Sciences and Research Committee, protocol number 941.

Provenance and peer review Not commissioned; externally peer reviewed.

Data sharing statement The authors are willing to share management protocols, data collection tools, data and any other relevant information associated with this observational study on demand.

Open access This is an open access article distributed in accordance with the Creative Commons Attribution Non Commercial (CC BY-NC 4.0) license, which permits others to distribute, remix, adapt, build upon this work non-commercially, and license their derivative works on different terms, provided the original work is properly cited, appropriate credit is given, any changes made indicated, and the use is non-commercial. See: http://creativecommons.org/licenses/by-nc/4.0/.

\section{REFERENCES}

1. Liu L, Oza S, Hogan D, et al. Global, regional, and national causes of child mortality in 2000-13, with projections to inform post-2015 priorities: an updated systematic analysis. The Lancet 2015;385:430-40.

2. Lazzerini M, Seward N, Lufesi N, et al. Mortality and its risk factors in Malawian children admitted to hospital with clinical pneumonia, 2001-12: a retrospective observational study. Lancet Glob Health 2016;4:e57-e68.

3. Kissoon N, Uyeki TM. Sepsis and the global burden of disease in children. JAMA Pediatr 2016;170:107.

4. Takem EN, Roca A, Cunnington A. The association between malaria and non-typhoid Salmonella bacteraemia in children in sub-Saharan Africa: a literature review. Malar J 2014;13:400.

5. Church J, Maitland K. Invasive bacterial co-infection in African children with Plasmodium falciparum malaria: a systematic review. BMC Med 2014;12:31.

6. Rimensberger PC, Cheifetz IM. Ventilatory support in children with pediatric acute respiratory distress syndrome. Pediatr Crit Care Med 2015;16:S51-S60.

7. Barry P, Morris K, Tariq A. Oxford specialist handbook: paediatric intensive care. Oxford University Press, 2010.

8. Davis AL, Carcillo JA, Aneja RK, et al. American college of critical care medicine clinical practice parameters for hemodynamic support of pediatric and neonatal septic shock. Crit Care Med 2017;45:1061-93.

9. McCollum ED, King C, Deula R, et al. Pulse oximetry for children with pneumonia treated as outpatients in rural Malawi. Bull World Health Organ 2016;94:893-902.

10. Duke T, Wandi F, Jonathan M, et al. Improved oxygen systems for childhood pneumonia: a multihospital effectiveness study in Papua New Guinea. The Lancet 2008;372:1328-33.
11. Bradley BD, Light JD, Ebonyi AO, et al. Implementation and 8-year follow-up of an uninterrupted oxygen supply system in a hospital in The Gambia. Int J Tuberc Lung Dis 2016;20:1130-4.

12. World Health Organisation. WHO technical specifications for oxygen concentrators. WHO, 2016.

13. Belle J, Cohen H, Shindo N, et al. Influenza preparedness in lowresource settings: a look at oxygen delivery in 12 African countries. J Infect Dev Ctries 2010;4:419-24.

14. Duke T, Hwaihwanje I, Kaupa M, et al. Solar powered oxygen systems in remote health centers in Papua New Guinea: a large scale implementation effectiveness trial. J Glob Health 2017;7:010411.

15. Essouri S, Carroll C. Noninvasive support and ventilation for pediatric acute respiratory distress syndrome. Pediatr Crit Care Med 2015;16:S102-S110.

16. Argent AC, Biban P. What's new on NIV in the PICU: does everyone in respiratory failure require endotracheal intubation? Intensive Care Med 2014:40:880-4.

17. Morley SL. Non-invasive ventilation in paediatric critical care. Paediatr Respir Rev 2016;20:24-31.

18. Essouri S, Chevret L, Durand P, et al. Noninvasive positive pressure ventilation: five years of experience in a pediatric intensive care unit. Pediatr Crit Care Med 2006;7:329-34

19. Essouri S, Durand P, Chevret L, et al. Optimal level of nasal continuous positive airway pressure in severe viral bronchiolitis. Intensive Care Med 2011;37:2002-7.

20. Essouri S, Laurent M, Chevret L, et al. Improved clinical and economic outcomes in severe bronchiolitis with pre-emptive nCPAP ventilatory strategy. Intensive Care Med 2014;40:84-91.

21. Gupta S, Donn SM. Continuous positive airway pressure. Clin Perinatol 2016;43:647-59.

22. Martin S, Duke T, Davis P. Efficacy and safety of bubble CPAP in neonatal care in low and middle income countries: a systematic review. Arch Dis Child - Fetal Neonatal 2014;99:F495-F504.

23. Chisti MJ, Salam MA, Smith JH, et al. Bubble continuous positive airway pressure for children with severe pneumonia and hypoxaemia in Bangladesh: an open, randomised controlled trial. The Lancet 2015;386:1057-65.

24. Wilson PT, Baiden F, Brooks JC, et al. Continuous positive airway pressure for children with undifferentiated respiratory distress in Ghana: an open-label, cluster, crossover trial. Lancet Glob Health 2017;5:e615-e623.

25. Walk J, Dinga $P$, Banda $C$, et al. Non-invasive ventilation with bubble CPAP is feasible and improves respiratory physiology in hospitalised Malawian children with acute respiratory failure. Paediatr Int Child Health 2016;36:28-33.

26. Lang H, Mlotha-Mitole R, Phiri A. ABSTRACT 34: reduction of paediatric hospital mortality in a malawian referral hospital. Pediatr Crit Care Med 2014;15:11-12.

27. WHO. Pocket book of hospital care for children : guidelines for the management of common childhood illnesses, 2013.

28. World Health Organization. Guideline: updates on paediatric emergency triage, assessment and treatment: care of critically-ill children, 2016.

29. Holliday MA, Segar WE. The maintenance need for water in parenteral fluid therapy. Pediatrics 1957;19:823-32.

30. McNab S, Duke T, South M, et al. $140 \mathrm{mmol} / \mathrm{L}$ of sodium versus $77 \mathrm{mmol} / \mathrm{L}$ of sodium in maintenance intravenous fluid therapy for children in hospital (PIMS): a randomised controlled double-blind trial. The Lancet 2015;385:1190-7.

31. Olson D, Preidis GA, Milazi R, et al. Task shifting an inpatient triage, assessment and treatment programme improves the quality of care for hospitalised Malawian children. Trop Med Int Health 2013;18:879-86.

32. Slater A, Shann F, Pearson G, et al. PIM2: a revised version of the paediatric index of mortality. Intensive Care Med 2003;29:278-85.

33. Pollack MM, Patel KM, Ruttimann UE. PRISM III: an updated pediatric risk of mortality score. Crit Care Med 1996;24:743-52.

34. Olson D, Davis NL, Milazi R, et al. Development of a severity of illness scoring system (inpatient triage, assessment and treatment) for resource-constrained hospitals in developing countries. Trop Med Int Health 2013;18:871-8.

35. Hooli S, Colbourn T, Lufesi N, et al. Predicting hospitalised paediatric pneumonia mortality risk: an external validation of risc and mrisc, and local tool development (RISC-Malawi) from Malawi. PLoS One 2016:11:e0168126.

36. Reed C, Madhi SA, Klugman KP, et al. Development of the Respiratory Index of Severity in Children (RISC) score among young children with respiratory infections in South Africa. PLoS One 2012;7:e27793 
37. Haniffa R, Isaam I, De Silva AP, et al. Performance of critical care prognostic scoring systems in low and middle-income countries: a systematic review. Crit Care 2018;22:18.

38. Smith ZA, Ayele Y, McDonald P. Outcomes in critical care delivery at Jimma University Specialised Hospital, Ethiopia. Anaesth Intensive Care 2013;41:363-8.

39. Dünser MW, Towey RM, Amito J, et al. Intensive care medicine in rural sub-Saharan Africa. Anaesthesia 2017;72:181-9.

40. Argent AC, Ahrens J, Morrow BM, et al. Pediatric Intensive Care in South Africa. Pediatr Crit Care Med 2014;15:7-14.

41. Esquinas Rodriguez AM, Scala R, Soroksky A, et al. Clinical review: Humidifiers during non-invasive ventilation - key topics and practica implications. Critical Care 2011;16:203.

42. Chidekel A, Zhu Y, Wang J, et al. The effects of gas humidification with high-flow nasal cannula on cultured human airway epithelial cells. Pulm Med 2012;2012:1-8.

43. Preidis GA, McCollum ED, Mwansambo C, et al. Pneumonia and malnutrition are highly predictive of mortality among African children hospitalized with human immunodeficiency virus infection or exposure in the era of antiretroviral therapy. J Pediatr 2011;159:484-9.

44. Smith AG, Eckerle M, Mvalo T, et al. CPAP IMPACT: a protocol for a randomised trial of bubble continuous positive airway pressure versus standard care for high-risk children with severe pneumonia using adaptive design methods. BMJ Open Respir Res 2017;4:e000195.

45. Oakley E, Borland M, Neutze J, et al. Nasogastric hydration versus intravenous hydration for infants with bronchiolitis: a randomised trial. Lancet Respir Med 2013;1:113-20.

46. Falk M, Donaldsson S, Drevhammar T. Infant CPAP for low-income countries: An experimental comparison of standard bubble CPAP and the Pumani system. PLoS One 2018;13:e0196683.

47. Schneider J, Khemani R, Grushkin C, et al. Serum creatinine as stratified in the RIFLE score for acute kidney injury is associated with mortality and length of stay for children in the pediatric intensive care unit. Crit Care Med 2010;38:933-9.

48. von Seidlein L, Olaosebikan R, Hendriksen IC, et al. Predicting the clinical outcome of severe falciparum malaria in african children: findings from a large randomized trial. Clin Infect Dis 2012;54:1080-90.

49. Conroy AL, Hawkes M, Elphinstone RE, et al. Acute Kidney Injury Is Common in Pediatric Severe Malaria and Is Associated With Increased Mortality. Open Forum Infect Dis 2016;3:ofw046.

50. World Health Organisation. Revised WHO classification and treatment of childhood pneumonia at health facilities. WHO, 2014. 